\title{
La santé
}

\section{haute déffinition}

\section{Autour de la notion \\ d'homme « augmenté »}

Florence Arnoux

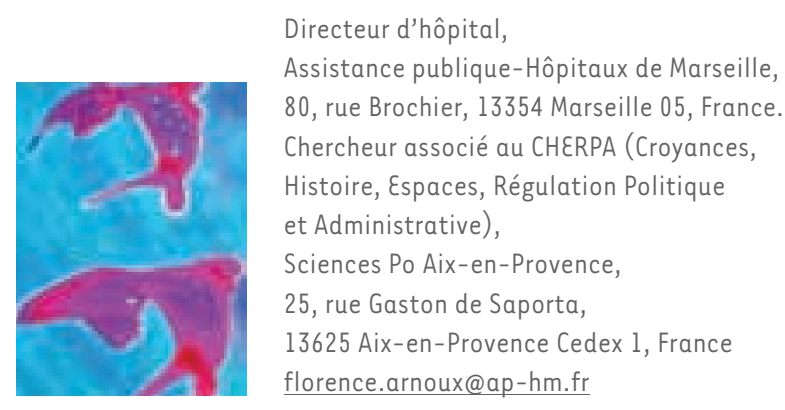

Technologies de la santé et mythe de l'homme « réparable »

Aujourd'hui encore, nous nous représentons essentiellement notre corps comme un organisme invariant, stable, circonscrit, où les yeux permettent de voir, les poumons de respirer... Comment imaginer ce que pourra demain un corps qui serait prolongé chimiquement, mécaniquement, informatiquement, un homme «augmenté » (enhanced man), amélioré par les technosciences ? Cette question intéresse de plus en plus les sciences biomédicales, captive les chercheurs et retient l'attention du grand public. Nous aspirons en effet tous à une meilleure santé, une vie dépourvue de douleur, de souffrance, à faire l'expérience d'un bien-être complet. À cette aspiration généralisée s'ajoute l'obsession de la société de remplacer l'homme au travail tout en le rendant plus efficace, plus productif, plus performant. En survalorisant les performances individuelles, nos sociétés post-industrielles encouragent en quelque sorte le dopage dans la pratique sportive, professionnelle, sexuelle, militaire. Enfin, et surtout, parmi la nébuleuse de désirs qui nous animent figure la volonté démiurgique de retarder l'échéance de la mort, de faire reculer la fatalité, de dépasser la frontière biologique programmée dans notre ADN et dite «limite de Hayflick ${ }^{1}$,

Ce texte rend compte des discussions qui ont eu lieu lors des sixièmes « Rencontres annuelles de la santé », organisées par F. Arnoux le 20 novembre 2009, à l'Institut d'études politiques d'Aix-en-Provence.

${ }^{1}$ Mse en lumière en 1965 par le Pr Leonard Hayflick, I'un des fondateurs de la biologie moderne du vieillissement.

du nom du microbiologiste américain qui démontra dès 1965 que les cellules ne pouvaient se diviser qu'un nombre fini de fois avant de mourir. Rompre avec l'entropie, soustraire l'homme au deuxième principe de la thermodynamique: de très sérieux scientifiques, comme ceux qui officient dans le très célèbre Massachusetts Institute of Technology (MIT), s'y essayent.

Les possibilités d'intervention sur l'homme sont grandes aujourd'hui et les technologies de la santé, qu'elles soient préventives, diagnostiques ou thérapeutiques, ont beaucoup évolué sous les effets des progrès, entre autres, de la biologie, de la physique, de la chimie et de l'informatique. La convergence des biotechnologies actuelles, des neurosciences, de la biologie de synthèse, de la prothétique, de la robotique, des technologies de l'information, des nanotechnologies permet de franchir les frontières connues ou de créer des interfaces entre les espèces (humaine, végétale, animale), entre les genres (hommes-femmes), entre l'homme et l'artéfact, entre l'homme et le divin, ou encore entre le vivant et l'inerte.

Si aujourd'hui on sait décupler la force, on ignore encore en revanche comment augmenter la créativité, l'intuitivité, l'oreille musicale, le gai savoir ${ }^{2}$ [1] que Nietzsche attribue au «surhomme $e^{3}$ ». Les résultats de la thérapie génique restent encore limités en termes d'impact

\footnotetext{
${ }^{2}$ F. Nietzsche, Euvres complètes, vol. II, Le Gai Savoir, Paris, Robert Laffont, 1993.

${ }^{3}$ F. Nietzsche, Ainsi parlait Zarathoustra. Prologue, § 3, Trad. de Maurice Betz, Paris,
} Le Livre de Poche, 1963. 
thérapeutique. De l'avis de Claude Bagnis", elle a plus alimenté le mythe de l'homme «réparable » que celui de l'homme «réparé » et il est regrettable que les orientations de la recherche fondamentale et appliquée soient dictées par les lois du marché. Mais, à n’en pas douter, les prochaines décennies verront la réalisation d'un certain nombre des promesses actuelles de la biologie moléculaire, de la génomique, du clonage ou encore de la médecine régénérative. Les secrets du génome humain commencent à être percés. La médecine a entamé sa personnalisation: des tests de détection de mutation des gènes permettent de déceler des prédispositions au cancer du sein ou au développement de certains mélanomes. Les biothérapies ouvrent des espoirs dans la prise en charge des cancers incurables. La thérapie génique combinée à la thérapie cellulaire donne des premiers résultats et il est probable qu'un jour prochain un grand nombre de maladies congénitales puisse être traité et prévenu.

\section{Un nouvel imaginaire}

Ces promesses de transformation profonde de l'homme, à la fois physique, biologique et génétique, qu'offrent la cybernétique, la bioingénierie, la thérapie génique sont si grandes qu'un nouvel imaginaire émerge, relayé par une iconographie, une littérature et une cinématographie obsédées depuis toujours par la mutation humaine. Hier animal outillé, l'être vivant du III millénaire pourra-t-il être encore considéré comme un homme? C'est cette mutation que les sixièmes Rencontres de la santé organisées à Sciences Po Aix en novembre 2009 avaient choisi d'examiner. Car s'il est un mot qui ne fait pas l'unanimité autour de lui, c'est bien celui d'«augmentation ». Un trouble collectif mal dissimulé entoure cette notion. Peut-être parce qu'elle éveille ou ravive un vertige moral face à toutes les possibilités et perspectives technoscientifiques qui s'ouvrent à nous et débordent notre cadre réflexif. La science progresse en effet désormais plus rapidement que la capacité humaine à concevoir ses avancées, à les appréhender par le vocable classique, plus rapidement que la conscience morale qui tente de la saisir. Comprendre cette mutation exige de réviser nos conceptions sociologiques, philosophiques et éthiques car ces progrès vertigineux modifient en profondeur nos représentations culturelles sur les notions de «soi », d'« Autre », d'identité, d'humanité, d'esprit, de conscience, d'âme, d'intention, de statut du corps humain, de dignité, d'intégrité, et permettent de nouveaux usages du corps et de nouvelles extensions de l'esprit.

Les frontières traditionnelles de l'organisation biologique n'en finissent plus de se brouiller. Ce qui est aujourd'hui considéré comme relevant de la normalité (une vision 10/10 par exemple) pourrait être considéré demain comme pathologique. II devient difficile de départir ce qui relève de la prévention, de la thérapeutique, de la réparation, de la correction, de la (ré)-habilitation, de l'amélioration. Certains d'entre nous disposent d'un organiseur électronique, d'un GPS, portent une montre, des lentilles de contact, des lunettes, un appareil dentaire,

${ }^{4}$ Directeur de recherche, Établissement français du sang. Participation à la table ronde «Réparer, transformer, augmenter ». prennent des vitamines, des bêtabloquants, font du sport, mangent cinq fruits et légumes par jour, boostent leur mémoire avec de l'huile de poisson ou en apprenant des poésies. Dirions-nous que ces pratiques nous aident à compenser ou corriger une fonction naturelle déficiente ou à améliorer notre nature (performance, résistance au stress, adaptabilité, capacités physiques, mémorielles, intellectuelles)? Ou les deux?

\section{Une « symbiose » humain-ordinateur}

Demain, grâce au progrès exponentiel des technologies appliquées sur le corps et le cerveau humain, nous recevrons peut-être des traitements adaptés à notre ADN, porterons une rétine artificielle ou un œil bionique, un exosquelette, hébergerons des puces-mémoire ou des neuroprothèses intelligentes, choisirons le sexe, la taille et les autres traits génétiques de nos enfants et serons peut-être vaccinés, en fonction de notre profil génétique, contre l'hypertension, tel ou tel cancer, la maladie d'Alzheimer ou encore certaines maladies auto-immunes. Peut-être même que des guerriers invincibles rempliront les rangs des armées. D'ores et déjà le soldat du présent, précurseur du guerrier du futur, est augmenté lorsqu'il a la connaissance de son environnement de combat, comme le démontre Marc Tanti $^{5}$, faisant un parallèle historique entre les techniques d'augmentation (depuis le gilet pare-éclats aux lunettes de vision nocturne) et les différentes épidémies du début du siècle jusqu'à aujourd'hui. Ainsi, pour anticiper sur les risques sanitaires auxquels pourrait être exposé le militaire, le préserver et augmenter ses capacités opérationnelles, le Service de santé des armées français a développé une mission de veille sanitaire de défense reposant essentiellement sur les technologies de l'information et de la communication. Mais les avancées en la matière ne s'arrêtent pas là : aujourd'hui déjà, nombre de laboratoires de sciences cognitives et programmes militaires (comme celui de la DARPA $^{6}$ ) explorent le domaine de la «cognition augmentée » qui se propose de créer une «symbiose» humain-ordinateur afin d'optimiser le fonctionnement du cerveau.

Que concocte-t-on dans les chaudrons de la médecine thérapeutique, curative ou palliative? Et dans ceux de la médecine artificialiste, de la performance

\footnotetext{
${ }^{5}$ Chercheur en sciences de l'information et de la communication, département d'épidémiologie et de santé publique, Institut de médecine tropicale du Service de santé des armées. Intervention sur « Mieux prédire le risque sanitaire pour le militaire en opération : l'enjeu du système de veille sanitaire de défense ».

${ }^{6}$ Defense advanced research projects agency, qui est une agence de la Défense
} américaine chargée des projets de recherche avancée à usage militaire. 
et du dépassement? Aujourd'hui, grâce aux implants cochléaires, les personnes atteintes de surdité comprennent $80 \%$ des mots prononcés et neuf phrases sur dix. Des pacemakers gèrent le rythme du cœur, des pompes à insuline adaptent la glycémie des diabètiques. La neurostimulation donne des résultats spectaculaires chez les patients atteints de la maladie de Parkinson. Mais pas seulement. Des chercheurs en neurosciences, comme Régine et Jean-Pierre Roll ${ }^{7}$, ont démontré, par leurs travaux récents, qu'il était possible d'animer virtuellement le corps pour le réparer: nos muscles renseignant notre cerveau sur nos mouvements, il «suffit» de stimuler des capteurs sensoriels pour créer l'illusion de mouvement des membres ou du corps dans son ensemble. Le courant qui nous pousse vers la mécanisation est fort. Les chirurgiens prothétiques $^{8}$, qu'ils s'occupent de mouvement ou de vision, témoignent d'avancées spectaculaires dans leurs domaines. La technologie du laser, que ce soit dans le domaine de la correction ophtalmologique ou dans celui de la thérapie photodynamique du cancer, a fait ses preuves. Les prothèses, orthèses et implants corrigent des invalidités. De nouveaux matériaux contribuent à étendre les possibilités d'emploi de ces techniques. Et nous avons tous en tête l'athlète sudafricain Oscar Pistorius qui, appareillé des deux jambes depuis l'enfance et triple médaillé d'or des Jeux paralympiques en 2008 , est considéré comme bénéficiant d'un avantage physique net grâce à ses prothèses en carbone. Ce qui fait dire à Jean-Michel Besnier ${ }^{9}$ que l'homme diminué peut devenir un homme augmenté. Reprenant à son compte cette idée de corps diminuéaugmenté, Jacques Brunet Georget ${ }^{10}$ cite l'exemple des transsexuels qui recherchent à conformer leur corps à leur «étantité » pour assurer une correspondance entre leur sexe anatomique et leur conviction profonde en tant que sujet. Ce « dépassement» du corps par la technique met alors en jeu la définition même de l'humain mais aussi et surtout requiert de penser de nouvelles réponses cliniques face aux nouvelles configurations du vivant.

\footnotetext{
${ }^{7}$ Respectivement ingénieur de recherche en neurosciences et professeur de neurosciences, Université de Provence-CNRS. Intervention sur «Animer virtuellement le corps pour le réparer : de la réalité à la fiction ».

${ }^{8}$ Jean-Noël Argenson, praticien hospitalo-universitaire en chirurgie orthopédique, responsable de l'Institut du mouvement, et Bernard Ridings, praticien hospitalouniversitaire en chirurgie ophtalmologique, Assistance publique-Hôpitaux de Marseille. Participation à la table ronde : «Réparer, transformer, augmenter ».

${ }^{9}$ Professeur des universités en philosophie, Université Paris-Sorbonne (Paris IV) et chercheur au CREA. Intervention sur «D'où vient que les perspectives d'« augmenter » I'homme éveillent le soupçon voire la frayeur?»

${ }^{10}$ Doctorant et chargé d'enseignement en philosophie, Université Bordeaux III. Intervention sur «Transsexualisme et corps prothétique: le «sexe» haute définition».
}

\section{L'homme augmenté, un rêve doublé d'un fantasme}

Les auteurs de romans d'anticipation, tels que George Orwell et Philippe K. Dick, annoncent depuis longtemps l'avènement prochain d'une forme d'intelligence supérieure à l'homme, d'une nouvelle entité. Car, pour reprendre une formulation de Gilles Deleuze et Félix Gattari, nous sommes tous des «machines désirantes » ${ }^{11}$. Plusieurs désirs nous animent dans notre course pour repousser et dissoudre les limites. À la suite de Leroi-Gourhan, le romancier d'anticipation

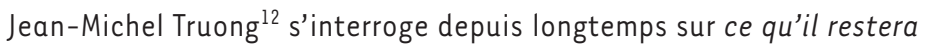
de l'homme après que la technique aura tout imité, après des millions d'années. La nostalgie de l'idée d'homme? II fait le pari qu'il demeurera plus que cela, une irréductibilité inimitable. Car tant qu'il y aura du récit, il y aura «de l'homme», quelle que soit la forme de cet être hypothétique, aussi dilué soit-il, voire sorti de l'impasse de la chimie du carbone. L'idée d'un homme augmenté, c'est donc d'abord un rêve doublé d'un fantasme. Un rêve de romancier de science fiction, de réalisateur de cinéma (de David Cronenberg à Spielberg), un rêve de biologiste (Pasteur), un rêve d'enfant qui voudrait devenir aussi fort qu'un super héros de bande dessinée, un rêve de scientifique (Stephen Hawking qui prône l'augmentation de l'homme pour faire face à la concurrence croissante des machines), un rêve d'idéologue (mouvements transhumaniste, extropien, singularitarien, etc.), un rêve d'alchimiste.

Cet imaginaire empreint de récits mythiques à base d'hybrides, d'êtres merveilleux, de récits des origines (mythe de l'Eden) est foisonnant et nous emmène bien loin des frontières et des interdits. Gommant la séparation entre humain et non humain, entre naturel et artificiel, il postule l'avènement d'une ère post-biologique, post-organique, et pourquoi pas post-humaine où le virtuel devient une extension du réel, réinventé, où la liberté morphologique et la liberté cognitive priment; où la réalité même devient augmentée.

Mais évidemment, et c'est bien naturel, nous oscillons entre fascination et peur devant le possible démantèlement de notre corps longtemps considéré comme sacré. Les éthiciens d'aujourd'hui, sous l'étendard des lois bioéthiques, ou encore de la Convention d'Oviedo sur les droits de l'Homme et de la biomédecine, qui vient de fêter cette année son $10^{e}$ anniversaire, - mais que la France n'a pas ratifiée, rappelons-le -, appellent à la plus grande vigilance, à l'autolimitation, et se refusent à être les témoins de l'effondrement de la tradition humaniste. Mais le discours éthique est-il apte à accompagner l'avènement d'un homme-réseau, homme-machine, au corps ou à l'enveloppe libéré de tout récit traditionnel? Selon Raphaël Liogier ${ }^{13}$, l'homme est une institution en péril, attaqué en tant qu'être moral, vidé de sa substance comme sujet, déconstruit à chaque blessure narcissique que le développement des scien-

\footnotetext{
${ }^{11}$ Ils y consacrent un chapitre (I) dans leur Anti-CEdipe, Paris, Éditions de Minuit, 1973.

${ }^{12}$ Romancier, essayiste et expert en intelligence artificielle. Intervention sur «Que restera-t-il de I'homme après que la technique aura tout imité au mieux? »

${ }^{13}$ Professeur des universités (sociologue et philosophe), directeur de l'Observatoire du religieux. Intervention sur «Construction, reconstruction, déconstruction de l'Homme»; il a coordonné le dossier De l'humain. Nature et artifices, de la revue La Pensée de Midi ( ${ }^{\circ} 30$, mars 2010).
} 
ces a infligée à l'humanitél4. Une éthique à trois visages, social d'abord, bioéthique ensuite et humanitaire enfin, est venue à son secours pour le reconstruire à travers des arguments philosophiques, anthropologiques, scientifiques. Mais - et c'est là la nouveauté observée par le sociologue - ces arguments, aussi rationnels soientils, n'emportent qu'une adhésion intellectuelle superficielle sans réussir à eux seuls à constituer l'adhérence mythique nécessaire à toute éthique vécue. Or les hommes ont besoin de sentir, d'éprouver, de rêver leur rôle, leur vie, de se raconter, avant toute discussion rationnelle sur les fins collectives. Sans cette croyance intime, il n'y a pas d'éthique efficace possible. Ce qui n'empêche pas, après le récit, provoquant l'adhérence des désirs humains, de chercher l'adhésion rationnelle fondée scientifiquement. C'est d'ailleurs la force du transhumanisme $e^{15}$ de jouer sur les deux champs ${ }^{16}$.

\section{Le choix de redevenir homme}

Cette promesse de surhomme a de quoi nous inquiéter, voire effrayer. La politique d' «amélioration» qui a conduit le régime nazi à la « solution finale », c'est-à-dire à l'extermination de millions d'êtres humains dont le seul tort était de ne pas appartenir à la race aryenne, est encore dans les esprits. Certains d'ailleurs s'inquiètent des conséquences de ces transformations génétiques sur la démocratie et la civilisation en général, comme le politologue américain Francis Fukuyama qui parle avec inquiétude d'un avenir «post-humain » qui pourrait devenir inhumain ${ }^{17}$.

Rejoignant cette idée que le récit nous caractérise et fait de nous ce que nous sommes aujourd'hui et ce que nous sommes en train d'advenir, Jean-Michel Besnier s'est essayé à dépeindre pourquoi ces perspectives d'augmentation suscitent le soupçon voire la frayeur. Penseur de longue date des utopies post-humaines, il relève un paradoxe essentiel : puisque l'augmentation nous rapproche d'une «santé parfaite $»^{18}$, pourquoi cette idée est-t-elle éconduite voire rejetée? Pour des raisons tout à la fois sociopolitiques (la peur de la scission en deux de l'humanité), culturelles (le culte de la transcendance et de l'humilité auquel doit se ranger l'homme) et métaphysiques (notre relation à l'autre étant conditionnée, commandée par notre fragilité, notre finitude) nous dit-il.

Après la recherche de la singularité portée par le courant transhumaniste, Besnier postule que les post-humains tenteront de retrouver leur vulnérabilité d'autrefois en faisant le choix de redevenir

${ }^{14}$ Cf. S. Freud, Introduction à la psychanalyse, Paris, Payot, 1975, p. 266-267. Ces trois blessures narcissiques sont l'héliocentrisme (la terre n'est pas au centre de l'univers), l'évolution (I'homme n'est qu'une branche du règne animal) et la psychanalyse (l'homme, doté d'un inconscient, n'est pas totalement maître de ses agissements).

${ }^{15}$ Selon ce courant d'idée alternatif, l'individu doit passer par plusieurs étapes : en premier lieu celle de sa transformation biochimique; ensuite celle de son amélioration via les nanotechnologies, et, passées ces deux étapes, il atteindra la singularité, où il pourra être éternellement réparé, avec transfert de mémoire d'un corps à l'autre.

${ }^{16}$ R. Liogier, «La vie rêvée de l'homme», La Pensée de Midi, n³0, mars 2010: De l'humain. Nature et artifices.

${ }^{17}$ F. Fukuyama, Our Posthuman future, Consequences of the Biotechnology Revolution, New York, Picador, 2003.

${ }^{18}$ L. Sfez, La Santé parfaite, Paris, Seuil, 1995 homme. Cette thèse rejoint celle du philosophe allemand Peter Sloterdijk, qui entrevoit une plus grande humanisation via l'anthropotechnie, ou art de la transformation de l'homme par lui même ${ }^{19}$. Empruntant à la pensée de Nietzsche et de Camus, Jean-François Mattéi ${ }^{20}$ s'interroge sur l'existence même de la nature humaine. Si le «premier homme» de Camus est un commencement, originellement sans racines, qui part à la recherche de son père, le «dernier homme» de Nietzsche erre et s'éloigne toujours de son origine, sombrant dans un nihilisme et un bien-être tiède. C'est ce spectre du dernier homme, désabusé, programmé, incapable de se raconter à travers un mythe fondateur que font planer les NBIC (nanotechnologies, biologie synthétiques, informatique et cognition) et les idéologies qui les entourent. Cet animal social en devenir serat-il capable de reconquérir son humanité, de redevenir homme, comme le prophète persan Zarathoustra après avoir parlé à l'astre solaire?

Au-delà de cette interrogation philosophique et ontologique, toutes ces perspectives requièrent une révision de nos conceptions économiques car il y a fort à parier que nous nous dirigeons, du moins dans l'horizon visible, vers un système uniquement accessible à une petite élite, en l'occurrence celle des pays les plus riches. Déjà, le coût actuel des biothérapies, des prothèses de dernière génération est exorbitant, à tel point que certains pays, comme la Suède, ne prennent plus en charge le traitement des biothérapies anticancéreuses des personnes ayant dépassé un certain âge. La question se pose alors de savoir s'il est de la vocation d'un système de protection sociale de prendre en charge le coût de ces modalités d'augmentation de l'homme. II semble difficile d'imaginer que le droit à la protection de la santé, universel, théoriquement, pourra comprendre, aux deux sens du terme, un droit à l'augmentation. Or si la santé de demain c'est la santé augmentée, le glas du droit universel à la santé sonnera-t-il ?

Allons plus loin dans le temps. L'homme du III millénaire, transformé, biotique, biocompatible, normativement défini, interfacé viendra-t-il supplanter l'homo sapiens-faber-econonomicus devenu obsolète, dépassé ? Certains, les transhumanistes notamment, rêvent de la possibilité qui leur sera peut-être un jour offerte de télécharger informatiquement leur cerveau. Cette hypothèse offrirait à l'individu, mais aussi au patient en stade terminal, une immortalité virtuelle: mais alors qui garantirait l'absence de copie,

\footnotetext{
${ }^{19} \mathrm{P}$. Sloterdijk, Règles pour le parc humain, Paris, Éditions Mille et une nuits, 2000.

${ }^{20}$ Professeur émérite de philosophie politique, Université de Nice-Sophia Antipolis et Sciences Po Aix, intervention sur «Le premier ou le dernier homme».
} 
la sauvegarde, protègerait contre le téléchargement ? D'autres, tels que les roboticiens, annoncent le remplacement de l'homme par la machine, pour devenir un artilect (artificial intellect). Le siècle prochain sera-t-il celui des robots sapiens, des humains artificiels, des machines animées, ou encore des «corps sans organes ${ }^{21}$ ? À défaut de pouvoir répondre à ces

${ }^{21}$ A. Artaud, Pour en finir avec le jugement de Dieu, Paris, Gallimard (coll. Poésie), 2003, première édition 1974. Formule poétique reprise ensuite par Gilles Deleuze et Félix Guattari dans L'Anti-Grdipe, pour en faire un concept philosophique: Capitalisme et schizophrénie 1, L’Anti-Crdipe, Paris, Éditions de Minuit (coll. Critique), 1995 , p. 494. questions, nous pouvons au moins opérer avec certitude un constat: nous sommes les témoins d'une métamorphose. Celle de l'humanité, dont la nature est de se transformer, qui se débarrasse peu à peu de ses peaux superflues. $\diamond$

The health high definition

\section{CONFLIT D'INTÉRÊTS}

L'auteur déclare n'avoir aucun conflit d'intérêts concernant les données publiées dans cet article.

\section{TIRÉS À PART}

F. Arnoux

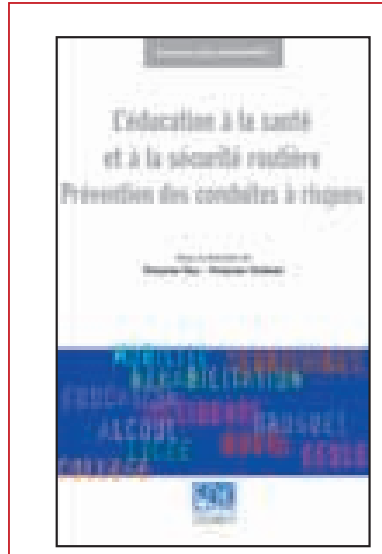

ISBN : 978-2-8425-4138-5 438 pages
Cntre Santé et Sécurité Routière, la prévention des risques liés à l'alcool et aux drogues Cmobilise les éducateurs. La cohérence dans l'application des plans ministériels successifs et programmes nationaux varie selon l'organisation et les pratiques territoriales: de nouveaux besoins émergent avec les changements de comportements individuels et les choix sociétaux de sécurité et de santé publiques. Entre chercheurs et acteurs, trois territoires contrastés (Ouest, Île-de-France, Est) sont observés : ils illustrent la complexité des réponses sanitaires, sécuritaires et sociales, qui fait écho à la complexité de l'étiologie des risques, des comportements plurifactoriels et des inégalités. Comment coordonner la diversité des statuts professionnels publics ou privés, comment allier les modèles développés par les chercheurs et les objectifs de prévention de proximité ? Cet ouvrage ouvre les perspectives de mutualisation des savoirs et des expériences.

\section{Françoise Facy - Françoise Chatenet}

À retourner à EDK, 2, rue Troyon - 92316 Sèvres Cedex

Tél. : 0155641393 - Fax : 0155641394 - E-mail : edk@edk.fr

NOM : Prénom :

Adresse :

Code postal :

Ville : Tél :

Pays :

Fonction :

Je souhaite recevoir l'ouvrage L'éducation à la santé et à la sécurité routière : $20 €+3 €$ de port $=\mathbf{2 3} € \mathbf{T T C}$

en ................ exemplaire, soit un total de $€$

$\square$ Par chèque, à l'ordre de $\mathbf{E} \mathbf{D}$ K

Par carte bancaire :

$\square$ Visa $\square$ Eurocard/Mastercard

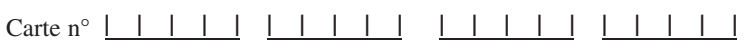

Date d'expiration: $\quad 1 \quad 1 \quad 1 \quad 1$

@ Date d'expiration : $\frac{||||}{N^{\circ} \text { de contrôle au dos de la carte : }}$

Signature : 


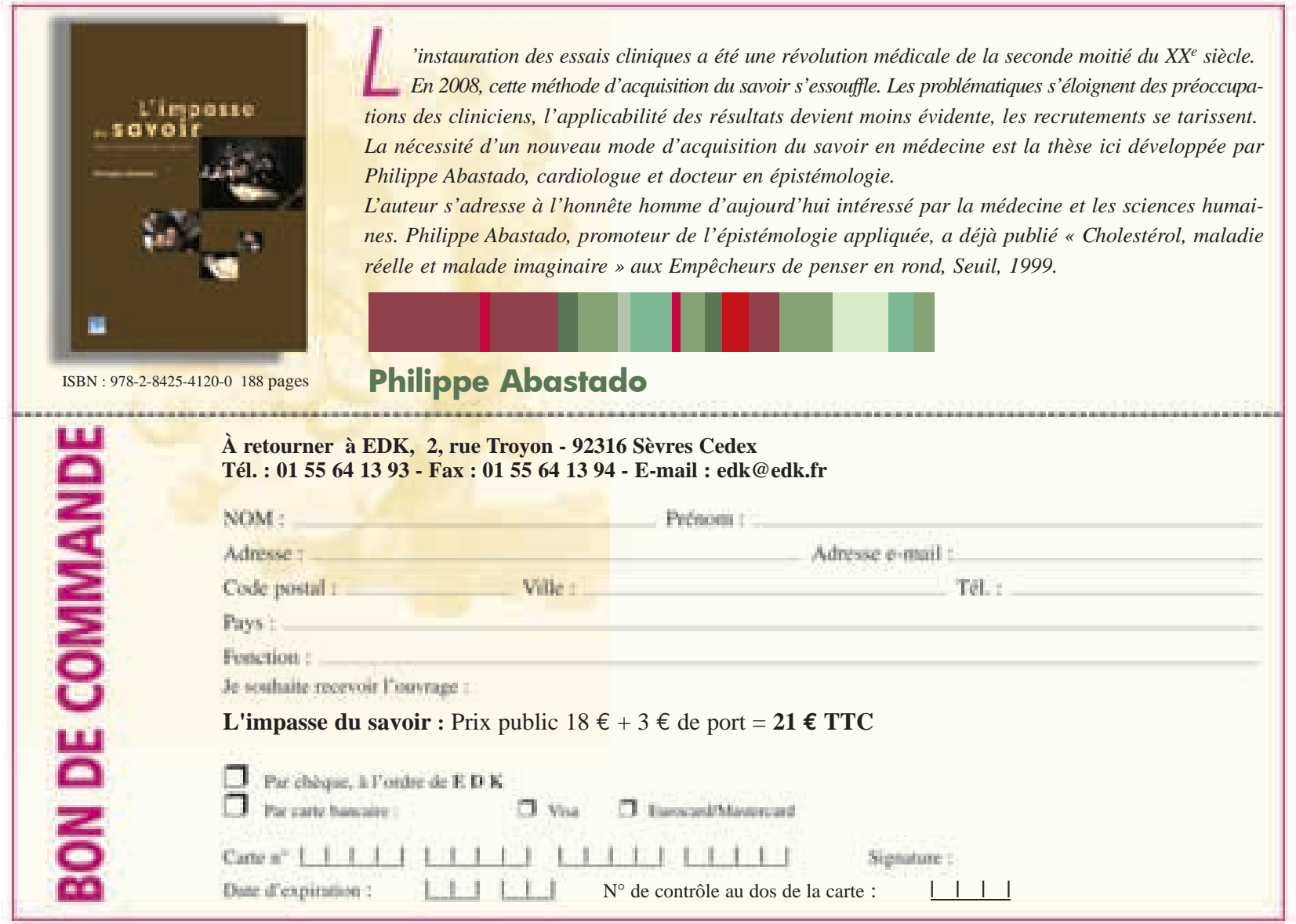

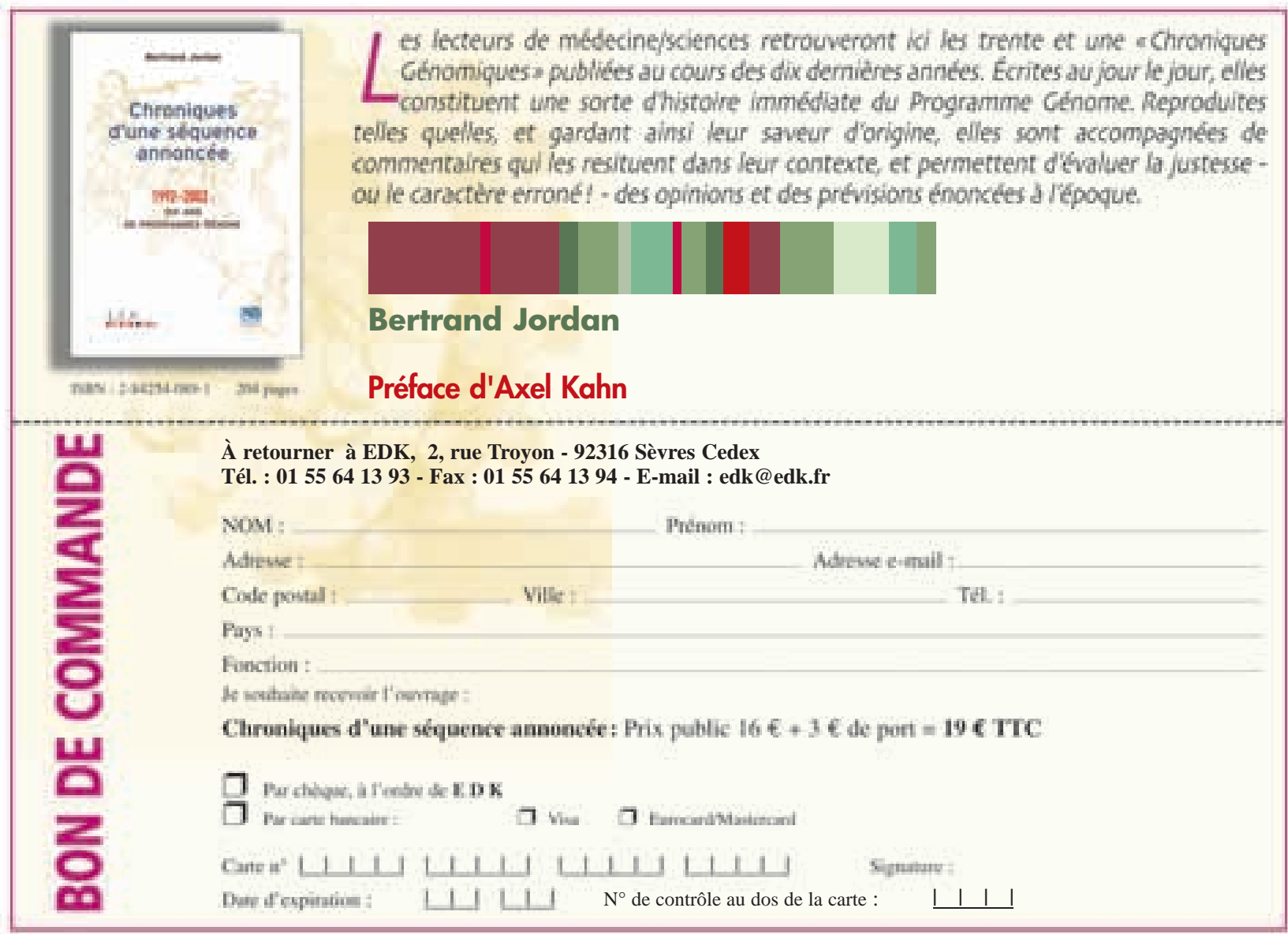

